\title{
Domestic EWH and HVAC Management in Smart Grids: Potential Benefits and Realization
}

\author{
Amir Safdarian*, Mubbashir Ali, Mahmud Fotuhi-Firuzabad, and Matti Lehtonen
}

Abstract - Demand response is envisioned as a key solution to increase network efficiency as well as to alleviate challenges such as growing demand. In this paper, domestic electric water heater $(\mathrm{EWH})$ and heating, ventilation, and air-conditioning (HVAC) are considered for potential demand response applications. An interaction between the system operator and consumers is considered to facilitate managing cyclic operation of consumers' heating loads. A centralized control model is first developed and formulated to achieve potential benefits of demand response of EWH and HVAC loads. Then, a distributed control algorithm is designed for practical implementation. In the proposed algorithm, house-wide modules (HwMs) submit their load modification proposals to system-wide module (SwM). SwM judges the received proposals and accepts those which are in line with the objective. This process iterates until no further proposals are received. Finally, the distributed control algorithm is applied to a group of consumers and the obtained results are thoroughly discussed. The objective is to attain a desirable load profile while the consumers' thermal comfort is not sacrificed. The analyses affirm significant benefits of demand response from domestic EWH and HVAC loads which are effectively realized by the distributed algorithm.

Keywords — demand response; distributed control; domestic consumer; EWH load; HVAC load

\section{INTRODUCTION}

Demand response enables power system operators to enhance power systems operation from sev-

* Amir Safdarian is with Department of Electrical Engineering, Sharif University of Technology, Tehran, Iran (Email: safdarian@ sharif.edu). 
eral aspects [1]. Demand response flattens load profiles thereby reduces network losses [2], prevents operation of high-cost/emission generating units [3]-[4], and defers capital intensive reinforcements [5]. Moreover, it is a promising complement to renewable energy resources with inherently variable generation [6]-[7]. These benefits have motivated electric companies around the world to make plans for activating demand response potential. Although majority of the implemented demand response programs have focused on large industrial consumers [8], substantial research has been devoted to activate demand response potential of domestic consumers. A research reported in [2] revealed that majority of residential demand response potentials can be unleashed by just managing the EWH and HVAC loads. This unique capability of EWH and HVAC loads is due to their significant contribution in total electricity usage as well as their great flexibility thanks to thermal inertia of building structures and heat storage capability. In Finland alone, thermal demand of a typical household accounts for about $70 \%$ of the total energy usage [9]. To this end, the aim of this paper is to study demand response potential of residential EWH and HVAC loads. Also, the paper presents a distributed management approach to bridge the gap between demand response potential and realization.

In order to clarify unique contribution of this paper, a survey on reported researches on demand response from domestic EWH and HVAC loads and the relevant management approaches is given here. The research reported in [10] has presented an algorithm for managing operation of domestic EWH load to minimize energy expenses while consumer's comfort preferences remain intact. The model presented in [11] jointly optimizes the energy scheduling of electric vehicle and HVAC load in a house to minimize energy payments. In [12], operation of HVAC load and partial thermal storages has been optimized in the hope of reducing energy costs without sacrificing user's comfort. While most of the works focused on benefits to a single consumer, the system- 
wide benefits are envisioned to be significant as well. The work presented in [13] has estimated demand response potential of EWHs as a balancing reserve. The potential of HVAC loads in providing load balancing services was evaluated in [14]. The study demonstrated that demand response capability of HVAC loads varies significantly with temperature settings and outdoor temperatures. A centralized control mechanism of HVAC loads was proposed in [15] to provide load balancing services. A comparison between centralized and decentralized demand response frameworks has been conducted in [16] demonstrating the importance of developing decentralized management models. In [17]-[18], decentralized management approaches were developed to coordinate demand response from different customers. However, optimal operation cycling of EWH and HVAC loads was not considered in the reported researches. In [19], a control strategy was developed to dispatch HVAC loads such that variation of nonrenewable power demand is minimized. The research reported in the reviewed papers is valuable, as they focused on the capability of demand response in load balancing. This is why demand response from domestic EWH and HVAC loads can be used as a feasible tool to modify system load profile thereby enhancing power system operation from several aspects.

The research scope of this article is the thorough quantification of potential benefits of responsive domestic EWH and HVAC loads to operation of distribution networks as well as the development of a system-wide management approach to achieve them. The major contributions of this paper are twofold. It firstly develops a mathematical model to optimally manage operating cycles of domestic EWH and HVAC loads such that a desirable system load profile is attained while consumers' comfort remains intact. The developed optimization problem accounts existing thermal models to consider the dynamic thermal behavior of consumers' space heating/cooling as well as their domestic hot water consumption. The thermal comfort of consumers is taken into 
account through imposing lower and upper bounds on the hot water and indoor temperatures. The developed model considers the stochastic nature of uncertain parameters such as hot water consumption via a scenario-based stochastic programming approach. In the approach, the constraints are written for all likely scenarios and the expected value of the objective over the scenarios is optimized. The second major contribution is to develop a distributed framework to solve the formulated problem for real world applications. In the developed framework, SwM and HwMs are independent agents whose interaction leads to the solution of the provided mathematical formulation. In the framework, the role of SwM is to coordinate the operation of HwMs such that the desirable system load profile is achieved. Whereas, HwMs are in charge of providing SwM with load modification proposals as well as ensuring consumers' thermal comfort. SwM iteratively receives HwMs' proposals and apply only the beneficial ones until no new proposal is received. The distributed algorithm respects consumers' privacy since they are not forced to broadcast their technical data as well as desirable indoor and hot water temperatures. In addition, congestion in communication infrastructure does not occur since data exchange between consumers and the program administrator is limited to consumers' and system load profiles. The capability of the distributed framework is demonstrated via applying to a system with a group of domestic customers.

\section{Domestic EWH And HVAC MAnagement Model}

This paper considers a smart grid with a system operator serving multiple domestic consumers. It is assumed that the operator and consumers signed a contract allowing the operator to control operating cycle of EWH and HVAC loads without over riding the users' thermal preferences. The system operator needs a tool to effectively manage operating cycle of the loads. This section in- 
tends to present mathematical formulation of the problem of managing operating cycle of domestic EWH and HVAC loads to modify the system load profile. In the management model, the objective is to modify system load profile. This objective is considered since it is general enough to cover almost all load shaping situations such as peak clipping, valley filling, and load profile flattening. This is done through considering the desired load profile as input and minimizing the expected deviation of system load profile from the desired profile. It is clear that the desired profile is defined by the system operators according to their system, experiences, and preferences. For instance, if an operator encounters capacity shortage, it makes sense to focus on peak clipping strategies. In this case, the desired profile might be one whose peak can be met without violating capacity limits. As another example, in case the network losses minimization is the target, the desired profile can be a more even profile. The general objective considered in this model can be mathematically formulated as follows:

$$
\text { Minimize } A D=\sqrt{\sum_{s \in S} \sum_{t \in T} \pi_{s}\left(P_{t, s}-P_{t}^{d e s}\right)^{2}}
$$

Where, $t$ and $T$ are index and set of time intervals, respectively. $s$ and $S$ are index and set of likely scenarios, respectively. $A D$ stands for expected deviation of system load profile from the desired profile. $\pi_{s}$ is occurrence probability of scenario $s . P_{t, s}$ and $P_{t}^{\text {des }}$ represent actual value of system load at time $t$ and scenario $s$ and desired value of system load at time $t$, respectively. The system load is calculated by summing up individual consumers' loads as follows:

$$
P_{t, s}=\sum_{n \in N} P_{n, t, s}^{f i r m}+P_{n, t, s}^{h v a c}+P_{n, t, s}^{e w h} ; \quad \forall t \in T, \forall s \in S
$$

Where, $n$ and $N$ are index and set of consumers, respectively. $P_{n, t, s}^{\text {firm }}$ is power absorbed by firm loads of consumer $n$ at time $t$ and scenario $s$. In this paper, since the focus is on EWH and HVAC systems, all other appliances either flexible like dishwashers or inflexible like televisions 
are considered firm. $P_{n, t, s}^{\text {hvac }}$ and $P_{n, t, s}^{e w h}$ represent power absorptions of HVAC and EWH loads of consumer $n$ at time $t$ and scenario $s$.

The load management problem is subjected to a number of constraints preserving consumers' thermal comfort. The following constraints ensure that indoor and hot water temperatures are within their acceptable limits.

$$
\begin{gathered}
\underline{T_{n}^{i a}} \leq T_{n, t, s}^{i a} \leq \overline{T_{n}^{i a}} ; \quad \forall n \in N, \forall t \in T, \forall s \in S \\
\underline{T_{n}^{h w}} \leq T_{n, t, s}^{h w} \leq \overline{T_{n}^{h w}} ; \quad \forall n \in N, \forall t \in T, \forall s \in S
\end{gathered}
$$

In the above expressions, $T_{n, t, s}^{i a}$ and $T_{n, t, s}^{h w}$ are indoor and hot water temperatures, respectively,

associated with consumer $n$ at time $t$ and scenario $s . \overline{T_{n}^{i a}}$ and $\underline{T_{n}^{i a}}$ are upper and lower bounds of acceptable range for indoor temperature, respectively, for consumer $n . \overline{T_{n}^{h w}}$ and $T_{n}^{h w}$ are also upper and lower bounds of acceptable range for hot water temperature for consumer $n$, respectively. The acceptable ranges are defined by consumers according to their thermal preferences. It is clear that wider ranges provide the system operator with higher flexibilities while tighter ranges are favorable for those consumers whose thermal comfort is their first and foremost priority.

In order to model the HVAC load, there exist several thermodynamic models having some pros and cons. The complex theoretical models are more close to the real-world however cumbersome to implement in practice. Also, simplistic models do not yield realistic results. In order to achieve a fair trade-off between complexity and accuracy, the paper uses the 1-capacity model where the dynamics of indoor ambient temperature are captured using a lumped capacity representing building fabric heat capacity as well as air heat capacity. This selection is justified as the model has been frequently employed in academic research [20]-[21]. Interested readers are referred to [12] where the model subtleties are well elaborated. Using the model, indoor temperature is calculated 
as follows:

$C_{n} \frac{T_{n, t, s}^{i a}-T_{n, t-1, s}^{i a}}{\Delta t}=\eta_{n}^{h v a c} P_{n, t, s}^{h v a c}+H_{n}^{i o}\left(T_{t, s}^{o a}-T_{n, t, s}^{i a}\right)+H_{n}^{i g}\left(T_{t, s}^{g}-T_{n, t, s}^{i a}\right)+H_{n}^{i v}\left(T_{t, s}^{v}-T_{n, t, s}^{i a}\right) ; \quad \forall n \in N, \forall t \in T, \forall s \in S$

In (5), $C_{n}$ denotes thermal capacity of building of consumer $n . \Delta t$ is the length of each time interval. $\eta_{n}^{\text {hvac }}$ is efficiency coefficient of HVAC system associated with consumer $n . H_{n}^{i o}$ is the thermal conductance between indoor air and outdoor air. $T_{t, s}^{o a}$ represents outdoor ambient air temperature at time $t$ and scenario $s . H_{n}^{i g}$ is the thermal conductance between indoor air and ground. $T_{t, s}^{g}$ denotes ground temperature at time $t$ and scenario $s . H_{n}^{i v}$ is the thermal conductance between indoor air and ventilation air. $T_{t, s}^{v}$ denotes ventilation air temperature at time $t$ and scenario $s$. It is worthwhile to note that all parameters in (5) except indoor temperature and HVAC power absorption are either known or predicted. The indoor temperature and HVAC power absorption are among decision variables of the management model. It is worth mentioning that the two-capacity model does not consider some affecting parameters such as outdoor solar radiation. It is obvious that more complex models that can consider more affecting parameters could be used in this paper. However, the two-capacity model is selected since it is accurate enough and easy to understand and the focus of the article is concentrated on the developed system-wide coordination approach.

The EWH model used in this study is based on the well-known and well-accepted equivalent thermal parameter (ETP) model. Interested readers are referred to [22] where more detailed information about the model is given. Using the model, domestic hot water temperature can be calculated as follows:

$$
C_{w}\left(V_{n}-W_{n, t, s}\right) \frac{T_{n, t, s}^{h w}-\eta_{n}^{h w s} T_{n, t-1, s}^{h w}}{\Delta t}=\eta_{n}^{e w h} P_{n, t, s}^{e w h}-C_{w} W_{n, t, s} \frac{T_{n, t, s}^{h w}-T_{t, s}^{g}}{\Delta t} ; \forall n \in N, \forall t \in T, \forall s \in S
$$


In (6), $C_{w}$ is heat capacity of a unit volume of water. $V_{n}$ is volume capacity of the hot water storage associated with consumer $n . W_{n, t, s}$ represents hot water consumption of consumer $n$ at time $t$ and scenario $s . \eta_{n}^{h w s}$ and $\eta_{n}^{\text {ewh }}$ respectively denote efficiency coefficient of hot water storage and electric water heater. It is worth mentioning that all parameters in (6) except hot water temperature and EWH power absorption are either known or predicted. The hot water temperature and EWH power absorption are also among decision variables of the model.

In practice, HVAC systems can have discrete operation mode. This limit is taken into account through the following constraints:

$$
\begin{aligned}
P_{n, t, s}^{h v a c}= & \sum_{l_{\text {hvac }} \in L_{\text {hvac }}} b_{n, t, s, l_{\text {hvac }}}^{\text {hvac }} P_{n, l_{\text {hvac }}}^{\text {hvac }} ; \quad \forall n \in N, \forall t \in T, \forall s \in S \\
& \sum_{l_{\text {hvac }} \in L_{\text {hvac }}} b_{n, t, s, l_{\text {hvac }}}^{\text {hvac }} \leq 1 ; \quad \forall n \in N, \forall t \in T, \forall s \in S
\end{aligned}
$$

In (7)-(8), $l_{\text {hvac }}$ and $L_{\text {hvac }}$ are respectively index and set of working levels of HVAC systems. $b_{n, t, l_{\text {hvac }}}^{\text {hvac }}$ is a binary variable indicating whether HVAC of consumer $n$ is working at level $l_{\text {hvac }}$ at time $t$ and scenario $s$ or not. $P_{n, l_{\text {hvac }}}^{\text {hvac }}$ denotes power absorption rate of HVAC of consumer $n$ at working level of $l_{\text {hvac }}$. (8) ensures that HVAC of consumer $n$ at time $t$ and scenario $s$ is either off or operating in only one of its working levels. The following limits establish the characteristic of EWH systems:

$$
\begin{aligned}
& P_{n, t, s}^{e w h}=\sum_{l_{e w h} \in L_{e w h}} b_{n, t, s, l_{e w h}}^{e w h} P_{n, l_{e w h}}^{e w h} ; \quad \forall n \in N, \forall t \in T, \forall s \in S \\
& \sum_{l_{e w h} \in L_{e w h}} b_{n, t, s, l_{e w h}}^{e w h} \leq 1 ; \quad \forall n \in N, \forall t \in T, \forall s \in S
\end{aligned}
$$

Where, $l_{e w h}$ and $L_{e w h}$ are respectively index and set of working levels of EWH systems. $b_{n, t, s, l_{e w h}}^{e w h}$ is a binary variable expressing whether EWH of consumer $n$ is working at level $l_{\text {ewh }}$ at time $t$ and scenario $s$ or not. $P_{n, l_{e w h}}^{e w h}$ is the rated power of EWH of consumer $n$ at working level of $l_{\text {ewh }}$. 
In case EWH and HVAC systems can work at continuous power level, the above constraints are replaced by the following ones:

$$
\begin{gathered}
0 \leq P_{n, t, s}^{h v a c} \leq \overline{P_{n}^{h v a c}} ; \quad \forall n \in N, \forall t \in T, \forall s \in S \\
0 \leq P_{n, t, s}^{e w h} \leq \overline{P_{n}^{e w h}} ; \quad \forall n \in N, \forall t \in T, \forall s \in S
\end{gathered}
$$

Where, $\overline{P_{n}^{\text {hvac }}}$ and $\overline{P_{n}^{\text {ewh }}}$ are respectively the rating capacity of EWH and HVAC of consumer $n$. These expressions are to adhere rating capacity of EWH and HVAC systems.

As discussed earlier, the load management model aims to shape the system load to approach the desired profile. However, this may be achieved at the expense of imposing extra electricity usage to consumers. In order to avoid this situation, the following constraint ensures that total electricity consumption of each consumer at each scenario does not increase from its normal consumption when the load management model is applied.

$$
\sum_{t \in T} P_{n, t, s}^{f i r m}+P_{n, t, s}^{h v a c}+P_{n, t, s}^{e w h} \leq \sum_{t \in T} P_{n, t, s}^{b c} ; \quad \forall n \in N, \forall s \in S
$$

Where, $P_{n, t, s}^{b c}$ is the total electricity usage of consumer $n$ at time $t$ and scenario $s$ in the base case (without the load management model). It is worthwhile to mention that the system-wide coordination approach must not impose extra costs to customers. In such a case, (13) must be revised such that customers' energy cost is kept equal to or less than the cost before modifications. The following constraints are considered to guarantee that indoor and hot water temperatures obtained by the load management model for the last period of time are the same as those in the base case.

$$
\begin{array}{ll}
T_{n, t, s}^{i a}=T_{n, t, s}^{i a, b c} ; & \forall n \in N, \forall s \in S, t=|T| \\
T_{n, t, s}^{h w}=T_{n, t, s}^{h w, b c} ; & \forall n \in N, \forall s \in S, t=|T|
\end{array}
$$

Where, $T_{n, t, s}^{i a, b c}$ and $T_{n, t, s}^{h w, b c}$ are indoor and hot water temperatures for consumer $n$ and time $t$ and scenario $s$ in base case.

The management model may frequently switch on and off EWH and HVAC systems in order to 
achieve a more desirable system load profile. However, frequent switching actions reduce the life cycle of an electric appliance. In order to prevent frequent switching actions, the following constraints put a cap on the maximum number of switching actions:

$$
\begin{gathered}
\frac{b_{n, t, s}^{h v a c}-b_{n, t-1, s}^{h v a c}}{2}-\varepsilon \leq x_{n, t, s}^{\text {hvac }} \leq \frac{1+b_{n, t, s}^{\text {hvac }}-b_{n, t-1,}^{h v a c}}{2}+\varepsilon ; \quad \forall n \in N, \forall t \in T, \forall s \in S \\
\sum_{t \in T} x_{n, t, s}^{h v a c} \leq S_{n}^{h v a c} ; \quad \forall n \in N, \forall s \in S \\
\frac{b_{n, t, s}^{e w h}-b_{n, t-1,}^{e w h}}{2}-\varepsilon \leq x_{n, t, s}^{e w h} \leq \frac{1+b_{n, t, s}^{e w h}-b_{n, t-1, s}^{e w h}}{2}+\varepsilon ; \quad \forall n \in N, \forall t \in T, \forall s \in S \\
\sum_{t \in T} x_{n, t, s}^{e w h} \leq S_{n}^{e w h} ; \quad \forall n \in N, \forall s \in S
\end{gathered}
$$

Where, $x_{n, t, s}^{h v a c}$ and $x_{n, t, s}^{e w h}$ are binary variables indicating whether the respective appliance at the respective time and scenario is switched on. $S_{n}^{\text {hvac }}$ and $S_{n}^{\text {ewh }}$ are respectively maximum number of switching actions for EWH and HVAC systems of consumer $n$. It should be noted that $\varepsilon$ in the above formulas is a constant with an arbitrary small value which is used to linearize the product of binary variables.

The above optimization problem requires technical data of buildings, hot water storages, HVACs, and EWHs, outdoor, ventilation air, and ground temperatures, and consumers' hot water consumption as input parameters. Whereas, the indoor and hot water temperatures and operating cycles of HVACs and EWHs are decision variables. The problem is in a mixed-integer quadratic programming (MIQP) format which can be solved via commercially available solvers. It is worth mentioning that developing the above optimization model is the first major contribution of the paper since, to the best of authors' knowledge, the literature lacks the system-wide model that coordinates operation of domestic EWH and HVAC loads to deliver benefits to the system.

\section{Distributed MANAGEMENT Framework}

The preceding section provided mathematical formulation of a management model for domestic 
EWH and HVAC loads. The problem can be solved in a centralized manner where all required data including technical data of EWH and HVAC loads, thermal parameters of buildings and storages, and consumers' thermal preferences are gathered and synthesized in a control center. However, this may cause a barrier in enrollment of privacy conscious consumers who are unwilling to announce their preferences and habits. This may undermine benefits of the program. Besides, the huge data exchange needed in the centralized framework demands for more complex database systems and higher capacity communication infrastructures which are able to serve thousands of consumers. This may increase the implementation cost of the program. Moreover, size of the centralized problem grows as number of consumers increases. This may make the problem very difficult to be solved in networks with thousands consumers. This huge burden can crash the central decision making processors. To cope with the mentioned complexities raised due to the centralized control, a distributed management framework is presented in this section. The distributed framework assumes that each house is equipped with a module called HwM through which the system operator can monitor, control, and coordinate the operation and energy consumption of EWH and HVAC loads. HwMs receive information about current and favorable state of the system from a system-wide coordinator which is referred to as SwM. In response to the signals received from SwM, HwMs, on behalf of customers, make local decisions which are sent to SwM for its approval. The local decision is called proposal. SwM reviews the received proposals and approve them only if they are in line with its objective. Fig. 1 shows overall procedure of the distributed management framework whose steps are thoroughly explained below.

Step 1. Consumers set their desired hot water and indoor temperatures preferences. They also provide HwMs with their expected hot water consumption. These data constitute a portion of input data of the management framework. 
Step 2. Outdoor ambient and ground temperatures are among the input data of the framework. It is clear that the future value of these parameters needs to be forecasted. The temperatures can be forecasted by either HwMs or SwM. In case that SwM forecasts the temperatures, it has to announce them to HwMs.

Step 3. This step intends to calculate consumers' energy usage and hourly indoor and hot water temperatures in the base case when no system-wide load management framework is under use. In the base case, EWH and HVAC systems are not switched on (off) until the time a violation in indoor and hot water temperatures is observed. For instance, a working EWH continues to heat up the stored water until hot water temperature starts exceeding the upper bound of acceptable range. The value of parameters in the base case is required to ensure that the management framework does not impose any extra energy consumption to consumers. Once the parameters are calculated, HwMs send the obtained load profiles to SwM. At the end of this step, initialization of the management procedure is finished and system load profile modification is ready to begin.

Step 4. SwM aggregates the received load profiles to constitute the system load profile. Then, it announces the current system load profile along with its desirable profile to HwMs.

Step 5. Once current and desirable system load profiles are received by HwMs, they prepare their load reschedule proposal such that the current profile approaches to the desirable one without violating consumers' thermal preferences and imposing any extra energy consumption to them. This can be done by solving the following optimization problem:

$$
\text { Minimize } \quad A D=\sqrt{\sum_{s \in S} \sum_{t \in T} \pi_{s}\left(P_{t, s}^{n^{\prime}}+P_{t, s}^{n}-P_{t}^{d e s}\right)^{2}}
$$

Where, $P_{t, s}^{n^{\prime}}$ is the current system load at time $t$ and scenario $s$ minus the load associated with consumer $n$ at time $t$ and scenario $s . P_{t, s}^{n}$ can be calculated as follows: 


$$
P_{t, s}^{n}=P_{n, t, s}^{f i r m}+P_{n, t, s}^{h v a c}+P_{n, t, s}^{e w h} ; \quad \forall t \in T, \forall s \in S
$$

This optimization problem is subjected to (3)-(19) for consumer $n$. (3)-(4) principally ascertains the consumer thermal preferences. (5)-(6) are needed to calculate the indoor and hot water temperatures. (7)-(12) are associated with the technical limits of HVAC and EHW systems. (13) ensures that energy usage in the reschedule proposal does not exceed the base case consumption. Indoor and hot water temperatures associated with the last period of time obtained in the proposal scheme are forced to be the same as those in the base case using (14)-(15). Finally, (16)-(19) are to avoid frequent switching of EWH and HVAC systems. The solution of the above problem contains the consumer's usage profile. Note that the above optimization problem changes operation cycle of EWH and HVAC loads such that electricity usage profile is optimized while the input data set by consumers remain the same. This flexibility in the operation cycle of EWH and HVAC loads is mainly due to thermal inertia of hot water storage and building. Once the optimization problem is solved, if the obtained usage profile is in line with the desired system load profile, the HwM sends it to SwM as a load modification proposal. Otherwise, it is discarded. It is worth mentioning that HwMs solve the optimization problem in a synchronous manner, thus, the whole step takes a short time.

Step 6. SwM reviews the received HwMs' proposals and judges whether the system load profile approaches to the desirable one. This is done using a simple greedy algorithm. The greedy algorithm is an iterative optimization approach. The approach, in each iteration, searches the neighborhood of the last solution and makes the best local choice with the hope of finding a global optimum. Fig. 2 shows the judgment procedure using the greedy algorithm. As it can be seen in the figure, once a proposal is received, SwM checks whether the proposal is beneficial for the system load profile or not. If yes, the proposal is accepted; otherwise, it is rejected. SwM sends an 
approval message to HwMs whose proposal is accepted. It is worth mentioning that changes in system load profile due to accepted proposals is not concerning since there are usually thousands of customers with very little impact on total system load profile. In addition, the approach is iterative and customers with discarded proposal have the chance to improve the updated system load profile in the next iteration. Note that in this step, if no proposal is received, the load management process is terminated since no further improvement is possible.

Step 7. SwM updates usage profile of HwMs with accepted proposals and calculates the new system load profile. SwM releases the obtained system load profile and the load management procedure continues from Step 5.

The above distributed load management framework iteratively approaches system load profile to the desirable profile. It is worthwhile to mention that developing the above distributed framework is the second major contribution of the paper. In the framework, consumers' privacy is respected since decisions are made locally and consumers do not need to broadcast technical data of their loads as well as desirable indoor and hot water temperatures. In addition, congestion in communication infrastructure is avoided since data exchange between HwMs and SwM is limited to consumers' and system load profiles. Moreover, the greedy algorithm used to judge the received proposals is very simple and does not lead to crash in SwM's processors.

\section{NUMERICAL STUDY}

This section provides simulation results of a few study cases to demonstrate performance of the devised framework. As mentioned earlier, the developed framework aims at minimizing the expected deviation of the system load profile from the desired profile. The desired profile, as the input of the framework, is determined according to the system operators experiences and objec- 
tives. If the system encounters capacity shortages, the desired profile must be selected such that existing capacities can meet the system demand during peak periods. In case loss minimization is the target, the desired profile can be an even profile. If the system hosts renewable generation units, it makes sense to maximize the correlation between system load and variable generation. Without loss of generality, the desirable system load profile considered in the simulations is an even profile which can be calculated using a simple average function as follows:

$$
P_{t}^{d e s}=\frac{1}{|T|} \sum_{s \in S} \sum_{t \in T} \pi_{s} P_{t} ; \quad \forall t \in T
$$

It is worth noting that more even profiles are preferred by network operators since they lead to more effective utilization of networks. In general, flattening system load profile reduces network losses, alleviates voltage drops, enhances service reliability, and postpones likely reinforcements. Simulations are conducted on a group of 50 consumers in a typical day. In the simulations, without loss of generality, the stochastic nature of uncertain parameters is overlooked and the predicted values are considered as the only scenario. The parameters associated with the 1-capacity and ETP models used for modeling HVAC and EWH loads are respectively given in Tables I and II.

The time resolution in the simulations is set to 15 minutes. This means that a daily management horizon contains 96 time steps (i.e., 24 hours are equivalent to 9615 -minute time steps). Therefore, the optimization problem that is solved by HwMs contains about 480 continuous variables, 380 binary variables, and 28,000 constraints. The simulations are conducted in MATLAB software package while the optimization problems are solved using CPLEX solver in GAMS. Using a Quad Core 2.67 GHz processor, it takes less than 100 milliseconds to solve the optimization problem. Also, it takes a few milliseconds for SwM to judge each received proposal. This, besides the synchronously operation of HwMs, means that the developed framework finds the final solution in a few seconds. 


\section{A. Basic Results}

The following four cases are simulated to demonstrate the benefits of managing domestic EWH and HVAC loads and capability of the distributed framework in achieving them.

Case I: This case serves as a comparison benchmark against other cases to gauge the performance of the devised load management framework. This case presents a situation wherein no system-wide load management is taken in use. In this case, consumers' HVAC (EWH) systems are switched on once their indoor (hot water) temperature drops under the lower limit and switched off when it exceeds the upper limit.

Case II: This case simulates a condition in which the devised distributed framework is applied to establish a system-wide management of HVAC loads. Here, it is assumed that only $50 \%$ of all customers (i.e., 25 customers) are enrolled in the program and allowed the operator to cycle on/off their HVAC system. In this case, HwMs and SwM exchange data thereby optimize operating cycle of domestic HVACs such that system load profile is flattened. In this case, EWHs are switched on and off similar to Case I.

Case III: This case is similar to Case II but the framework is applied to optimize operating cycle of domestic EWHs. Here, it is assumed that only 50\% of all customers (i.e., 25 customers) are enrolled in the program and allowed the operator to cycle on/off their EWH system. In this case, HVACs are switched on and off like in Case I.

Case IV: This case simulates a condition in which the devised framework is to manage operating cycle of domestic HVACs and EWHs to flatten the system load profile. Here, it is assumed that only $50 \%$ of all customers (i.e., 25 customers) are enrolled in the program and allowed the operator to cycle on/off their HVAC and EWH systems.

The above cases are simulated and associated results are discussed hereinafter. Fig. 3 shows the 
total load profile for the group of consumers in Case I. In this figure, ETC represents consumption of all appliances except EWH and HVAC systems. As can be seen in the figure, this portion of load is not constant and change over time. The energy usage of appliances like dishwashers, televisions, and oven is considered as ETC which is considered inflexible and is the same in all of the four cases. It is to be noted that ETC load is referred to as firm load in the optimization problem.

As it can be seen in Fig. 3, the peak demand occurs in the evening while the load is much lower during midnight. The peak load happens at $9 \mathrm{pm}$ with its demand roughly 2.2 times larger than valley load at $1 \mathrm{am}$. The system load factor is $69.2 \%$ which is clearly far from an ideal load factor. According to the figure, EWH and HVAC loads have a significant contribution in demand within the peak period. EWH and HVAC loads respectively account for about $21 \%$ and $26 \%$ of the peak load. Fig. 4 shows the total load profile of the group of consumers in Case 2 where operation cycles of HVACs are coordinated to attain a smoother profile. As shown in this figure, a portion of HVAC load is shifted to off-peak periods which results in the load factor of $78.5 \%$. In this case, the peak demand of $184.259 \mathrm{~kW}$ occurs at $9 \mathrm{pm}$ while valley load of $120.413 \mathrm{~kW}$ happens at 1 am. The peak load is about $50 \%$ higher than the valley load. In this case, the share of EWH and HVAC loads in the peak demand is $9 \%$ and $29 \%$, respectively.

Fig. 5 depicts hourly load profile of the group of consumers in Case III wherein operation cycles of EWHs are managed to smoothen the profile. As it can be seen in the figure, a portion of EWH load is displaced from on-peak to off-peak periods. This leads to the load factor of $72.2 \%$. In this case, peak load of $199.796 \mathrm{~kW}$ happens at $10 \mathrm{pm}$ while valley load of $119.037 \mathrm{~kW}$ occurs at $2 \mathrm{am}$. This implies that the peak is roughly 1.7 times of the valley. In this case, EWH and HVAC loads respectively account for about $26 \%$ and $19 \%$ of the peak load at $10 \mathrm{pm}$. 
Fig. 6 depicts the obtained load profile in Case IV in which operation cycles of both HVACs and EWHs are coordinated. As it can be observed, the profile is much more uniform than those in the first three cases. In this case, peak and valley loads of $170.546 \mathrm{~kW}$ and $126.038 \mathrm{~kW}$ respectively occur at $10 \mathrm{pm}$ and 1 am resulting in the peak to valley ratio of about 1.4 . In this case, the contribution of EWH and HVAC loads is near $13 \%$ and $22 \%$ of the peak demand.

Fig. 7 compares the system load profiles obtained in the four cases. As it can be observed, more even profiles are achieved in Cases II, III, and IV when operating cycles of either or both EWH and HVAC systems are managed. It is also observed that the most even load profile is attained if operating cycles of both EWH and HVAC systems are collectively managed. Table III provides the major characteristics of the load profiles obtained in the four cases. As it can be seen from the results provided in the table, managing operating cycles of HVACs and EWHs by the developed algorithm improves the load profile characteristics. According to the results, the peak load in Case I decreases by $12.78 \%, 5.42 \%$, and $19.27 \%$ in Cases II to IV when the proposed algorithm is applied. The valley load is also increased by $25.87 \%, 24.44 \%$, and $31.76 \%$ in Cases II to IV, respectively. Finally, the load factor in Case I is increased by $13.42 \%, 4.38 \%$, and $20.71 \%$ in Cases II to IV, respectively. As expected, the improvements in Case IV in which both HVACs and EWHs are managed are much more significant as compared with Cases II and III in which either HVACs or EWHs are controlled. This much improvement in the load profile is attractive given the fact that about $25 \%$ of generation facilities and $10 \%$ of grid facilities in the U.S. are needed only during 400 hours of annual peak period [5].

In the proposed algorithm, (13) ensures that the system-wide coordination of HVACs and EWHs should not lead to a higher total electricity usage. For this reason, total energy usage in the four cases is calculated by summing up the hourly consumptions as shown in Table IV. As it can 
be observed, application of the developed algorithm reduces the total energy consumption. The total energy usage in Case I decreases by $1.1 \%, 1.3 \%$, and $2.6 \%$ in Cases II to IV, respectively. It is worthy to mention that the energy savings would benefit consumers as well by realizing savings in electricity bills. Note that this monetary saving is beside the incentive to be paid to consumers for participation in demand response control.

The above discussed modifications in the load profile would provide great benefits to different attributes of the operation of distribution networks. In order to quantify the benefits, the obtained load profiles are applied to a distribution network. The basic data associated with the network is given in [23]. The network, as shown in Fig. 8, feeds 61 distribution substations (20/0.4 kV) through two radial feeders with a normally open tie-line which can be closed when the network reliability is jeopardized. The load profiles associated with the four cases are applied to the network and power flow analysis is conducted to calculate system loading and voltage profiles as well as network losses. The power flow analysis is done via MATPOWER [24] which is an opensource package of MATLAB M-files. Fig. 9 shows the hourly worst bus voltage of the network for the four cases. It can be observed that application of the system-wide management framework in Cases II to IV would result in a more evenly distributed voltage profile with less severe voltage drops. As expected, the algorithm is more beneficial in Case IV rather than in Cases II and III. The most severe voltage drop in Case I is alleviated by $13 \%, 6 \%$, and $20 \%$ in Cases II to IV when the developed algorithm is used.

Table V compares the total network losses associated with the four cases. As it can be observed, application of the proposed algorithm leads to $4.15 \%, 4.06 \%$, and $7.6 \%$ reduction in the total network losses in Cases II to IV, respectively. The unique value of these reductions will be understood considering the fact that distribution network losses account for roughly $60 \%$ of total 
power system losses which are about 5-12\% of the total produced power [25].

The load modifications caused by the developed load management framework would enhance the service reliability during likely contingencies. In other words, the developed framework flattens load profile thereby reduces probability of involuntary load curtailments during emergency conditions. In order to quantify the benefits, a reliability analysis is conducted and indices are compared for the four cases. In the analysis, constituting the set of likely contingencies is followed by minimizing the cost of load curtailments during each likely contingency. The occurrence probability of the contingencies is combined with resulted load curtailments and costs to calculate reliability indices. The indices are system average interruption frequency index (SAIFI), system average interruption duration index (SAIDI), expected energy not served (EENS), and expected interruption cost (EIC). SAIFI and SAIDI respectively denote average number and duration of interruptions per consumer per day. EENS represents the expected energy curtailment caused by interruptions during the day. EIC is the expected damage cost caused by interruptions during the day. A detailed explanation on reliability assessment and how the indices are calculated can be found in [26]. Table VI gives the reliability indices for the four cases. As it can be seen, in Case IV, application of the proposed framework leads to roughly $3.8 \%$ and $4.5 \%$ enhancement in SAIFI and SAIDI, respectively. Also, EENS is reduced by $9.7 \%$ when EWH and HVAC loads are managed in Case IV. EIC is also reduced by $9.1 \%$ when operating cycles of the loads are coordinated in Case IV.

\section{B. Sensitivity Analyses}

\section{1) Penetration Level of Consumers}

In the simulations conducted in Section IV.A, it was assumed that 25 out of 50 consumers are enrolled in the program allowing the network operator to coordinate operation cycles of their 
HVACs and EWHs. However, in practice, consumers' willingness to enroll in such programs depends on several affecting parameters such as their standard of living and income. This may make consumers level of enrollment difficult to predict. In order to address this uncertainty, Case IV is simulated for different numbers of enrolled consumers and the obtained results are given in Table VII. As shown in this table, the results in all aspects are significantly improved as the penetration of enrolled consumers increases. As it can be seen, even if about $25 \%$ of EWHs and HVACs are controlled by the developed framework, peak load, load factor, network losses, and EENS are improved by $8.26 \%, 8.14 \%, 3.17 \%$, and $4.65 \%$, respectively. These improvements are increased to $28.58 \%, 33.53 \%, 12 \%$, and $15.24 \%$ when HVACs and EWHs of all consumers are controlled. These sheer benefits will serve as a strong motivation for operators to attract more consumers in demand response programs.

Finally, it is worth mentioning that, since in the developed framework, HwMs synchronously solve the devised problem, the run time does not substantially increase with the system size and number of enrolled consumers. This statement is justified through observations in the conducted simulations with different numbers of enrolled consumers.

\section{2) Allowed Temperature Deviation}

In the above reported studies, it was assumed that consumers' indoor temperature must be kept within $19^{\circ} \mathrm{C}$ to $23^{\circ} \mathrm{C}$ and their hot water temperature is bounded within $50^{\circ} \mathrm{C}$ and $60^{\circ} \mathrm{C}$. However, these bounds may vary among consumers. To investigate the impact of these parameters, Cases I and IV are simulated for different temperature bands and the achieved results are summarized in Table VIII. As it can be seen, applying the developed framework brings considerable improvements in studies with different allowed temperature bands. The key observation from the table is that wider ranges for allowed hot water and indoor temperatures lead to more enhancements. The 
least beneficial, but still significant, situation is associated with the study in which the narrowest temperature bands are applied. In case of narrow bands, limited flexibility in keeping the appliances on and off would lead to shallow improvements. Also, a narrow band leads to more frequent operation cycle but for shorter periods which, in turn, overwhelms benefits of operation management of the loads. A wider band, however, may lead to a great flexibility thereby resulting in more significant improvements.

\section{3) Outdoor Temperature}

In the above studies, outdoor temperature data was associated with a typical winter day in Finland. This section investigates the impact of outdoor temperature variation on performance of the developed framework. To do so, Cases I and IV are simulated for changes in outdoor temperature and the results are given in Table IX. As it can be seen, the achieved benefits remain significant by scaling outdoor temperature up or down. In case that outdoor temperature is not too cold, energy consumption of HVACs and EWHs is trivial and hence, managing their operation cycle is less beneficial. On the contrary, if outdoor temperature is very cold, HVACs and EWHs must be kept on most of the time which means the loads provide a trivial flexibility with less benefit.

Finally, it should be noted that there exist some other affecting parameters such as building technical data that can change the estimated benefit of coordinating demand response potential of domestic EWH and HVAC loads. However, since the conducted research aims at developing a distributed system-wide coordination method to achieve as much benefit as possible of the flexibility in the operation of domestic EWH and HVAC loads, sensitivity analyses on the other affecting parameters are not provided. 


\section{CONCLUSION}

This paper developed a distributed framework to iteratively coordinate operation cycle of residential consumers' HVACs and EWHs. SwM and HwMs, as building blocks of the framework, intend to flatten total load profile while maintaining consumers' thermal preferences. In each iteration of the framework, HwMs offer their load modification proposals among which SwM accepts those with positive impacts on the total load profile. This process iterates to the point at which no further improvement in the total load profile is possible. Performance of the framework was demonstrated by its application to a Finnish network serving a group of consumers. The simulation results suggest that by properly coordinating the operation cycle of HVACs and EWHs can bring considerable peak reductions. It has constructive impact on load factor, network losses, and service reliability as well. It was also demonstrated that these positive influences get stronger with the increase of penetration level of consumers in the program. Finally, it was observed that wider ranges for allowed indoor and hot water temperatures do not necessarily lead to more enhancements.

\section{REFERENCES}

[1] U.S. Federal Energy Regulatory Commission, National action plan on demand response, 2010.

[2] A. Safdarian, M. Fotuhi-Firuzabad, and M. Lehtonen, "Benefits of demand response on operation of distribution networks: a case study," IEEE Syst. Jour., to be published.

[3] A. Khodaei, M. Shahidehpour, and S. Bahramirad, "SCUC with hourly demand response considering inter temporal load characteristics," IEEE Trans. Smart Grid, vol. 2, no. 3, pp. 564571, Sep. 2011.

[4] S. Holland and E. Mansur, "Is real-time pricing green? The environ-mental impacts of electricity demand variance," Rev. Econ. Stat., vol. 90, no. 3, pp. 550-561, Aug. 2008.

[5] T. J. Lui, W. Stirling, and H. O. Marcy, "Get smart," IEEE Power Energy Mag., vol. 8, no. 3, pp. 66-78, May 2010.

[6] R. Sioshansi, "Evaluating the impacts of real-time pricing on the cost and value of wind generation," IEEE Trans. Power Syst., vol. 25, no. 2, pp. 741-748, May 2010. 
[7] C. Cecati, C. Citro, and P. Siano, "Combined operations of renewable energy systems and responsive demand in a smart grid," IEEE Trans. Sustain. Energy, vol. 2, no. 4, pp. 468-476, Oct. 2011.

[8] G. Barbose, C. Goldman, and B. Neenan, "A survey of utility experience with real time pricing," Ernest Orlando Lawrence Berkeley National Laboratory, Berkeley, CA, Tech. Rep. LBNL-54238, Dec. 2004.

[9] Statistics Finland [Online]. Available: www.stat.fi/til/index_en.html.

[10] P. Du, and N. Lu, "Appliance commitment for household load scheduling," IEEE Trans. Smart Grid, vol. 2, no. 2, pp. 411-419, Jun. 2011.

[11] D.T. Nguyen, and L. Le, "Joint optimization of electric vehicle and home energy scheduling considering user comfort preference,” IEEE Trans. Smart Grid, vol. 5, no. 1, pp. 188-199, Jan. 2014.

[12] M. Ali, J. Jokisalo, K. Siren, and M. Lehtonen, "Combining the demand response of direct electric space heating and partial thermal storage using LP optimization," Electr. Power Syst. Res., vol. 106, pp. 160-167, Jan. 2014.

[13] S. A. Pourmousavi, S. N. Patrick, and M. H. Nehrir, "Real-time demand response through aggregate electric water heaters for load shifting and balancing wind generation," IEEE Trans. Smart Grid, vol. 5, no. 2, pp. 769-778, Mar. 2014.

[14] N. Lu, "An evaluation of the HVAC load potential for providing load balancing service," IEEE Trans. Smart Grid, vol. 3, no. 3, pp. 1263-1270, Sep. 2012.

[15] N. Lu and Y. Zhang, "Design considerations of a centralized load controller using thermostatically controlled appliances for continuous regulation reserves," IEEE Trans. Smart Grid, vol. 4, no. 2, pp. 914-921, Jun. 2013.

[16] C. L. Siebert, et al., "Centralized and decentralized approach to demand response using smart plugs," IEEE PES T \& D Conference and Exposition, pp. 1-5, 2014.

[17] C. Gong, X. Wang, W. Xu, and A. Tajer, "Distributed real-time energy scheduling in smart grid: stochastic model and fast optimization," IEEE Trans. Smart Grid, vol. 4, no. 3, pp. 1476-1489, Sep. 2013.

[18] T. Chang, M. Alizadeh, and A. Scaglione, "Real-time power balancing via decentralized coordinated home energy scheduling," IEEE Trans. Smart Grid, vol. 4, no. 3, pp. 1490-1504, Sep. 2013.

[19] L. Zheng and L. Cai, "A distributed demand response control strategy using Lyapunov optimization," IEEE Trans. Smart Grid, vol. 5, no. 4, pp. 2075-2083, Jul. 2014.

[20] Z. Chen, L. Wu, and Y. Fu, "Real-time price-based demand response management for residential appliances via stochastic optimization and robust optimization," IEEE Trans. Smart Grid, vol. 3, no. 4, pp. 1822-1831, Dec. 2012.

[21] M. A. A. Pedrasa, T. D. Spooner, and I. F. MacGill, "Coordinated scheduling of residential distributed energy resources to optimize smart home energy services," IEEE Trans. Smart Grid, vol. 1, no. 2, pp. 134-143, Sep. 2010.

[22] 2008 ASHRAE Handbook-HVAC Systems and Equipment, ASHRAE Inc., 2008.

[23] http://sharif.edu/ safdarian/Domestic\%20EWH\%20and\%20HVAC.pdf

[24] R. D. Zimmerman, C. E. Murillo-Sánchez, and R. J. Thomas, "MATPOWER: steady-state operations, planning and analysis tools for power systems research and education," IEEE Trans. Power Syst., vol. 26, no. 1, pp. 12-19, Feb. 2011. 
[25] M.G. Lauby, "Reliability considerations for application of smart grid technologies," in Proc. IEEE PES GM, Minneapolis, MN, USA, Jul. 2010.

[26] R. E. Brown, Electric Power Distribution Reliability, 2nd ed. New York: CRC Press, 2009.

Figure captions:

Fig. 1. Flowchart of the decentralized approach.

Fig. 2. Judgment procedure of received proposals via greedy approach.

Fig. 3. Total load profile in Case I.

Fig. 4. Total load profile in Case II.

Fig. 5. Total load profile in Case III.

Fig. 6. Total load profile in Case IV.

Fig. 7. Total load profile in Cases I to IV.

Fig. 8. Single line diagram of the distribution network under study.

Fig. 9. Hourly worst bus voltages in Cases I to IV.

Table captions:

Parameters associated with the 1-capacity building model

Parameters associated with the ETP model

Characteristics of load profiles obtained in the four cases

Overview of total energy consumption in the four cases

Overview of total network losses in the four cases

Overview of reliability indices in the four cases

Impacts of the number of enrolled consumers on results in Case IV

Impact of allowed indoor temperature on benefits in Case IV

Impact of outdoor temperature on benefits in Case IV

Figures:

Fig. 1 


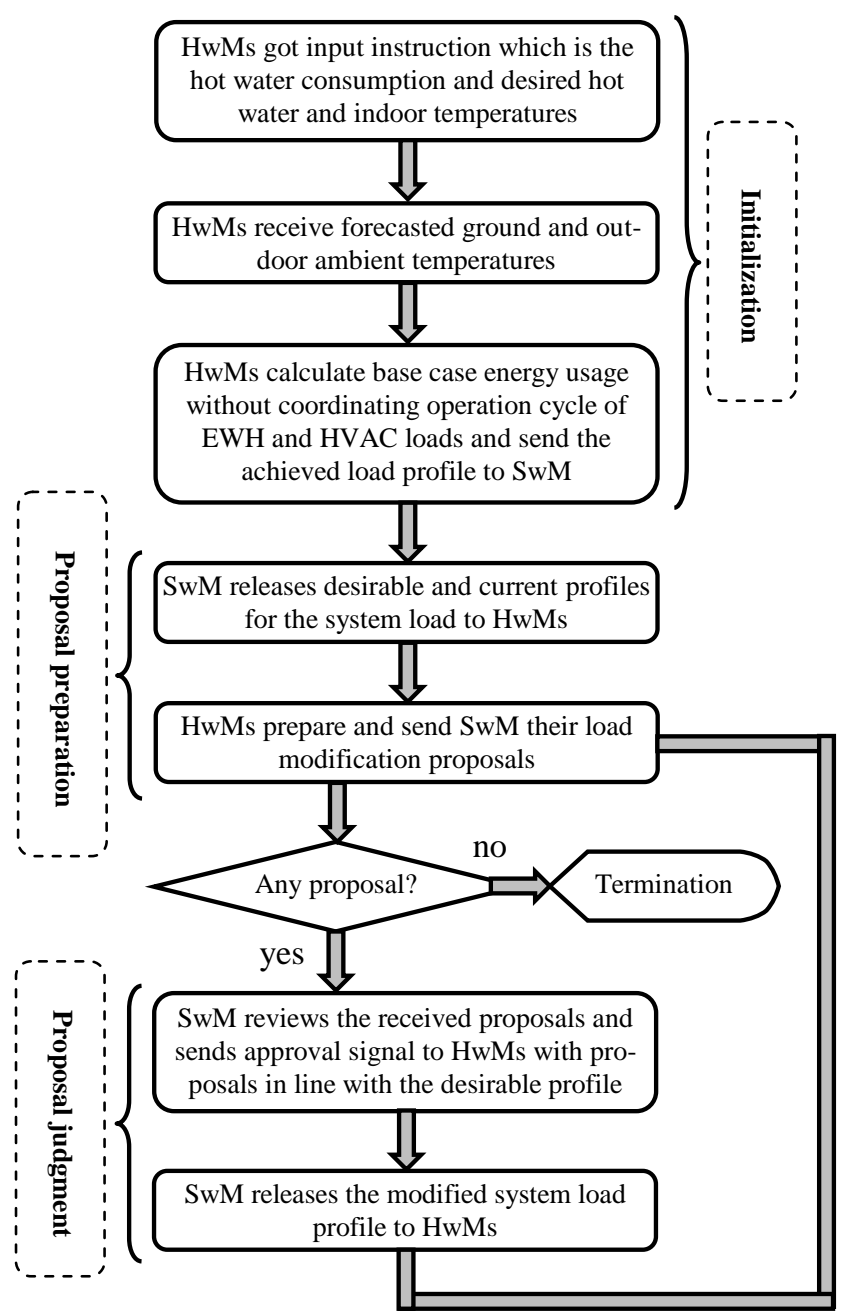

Fig. 2

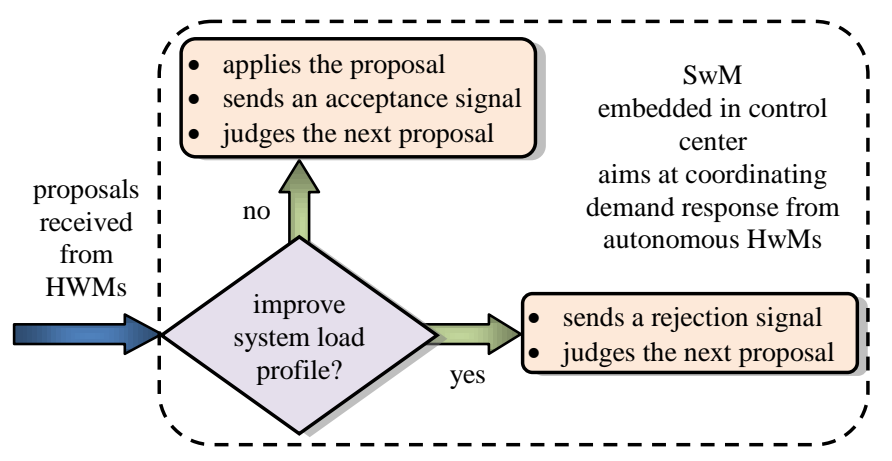

Fig. 3 


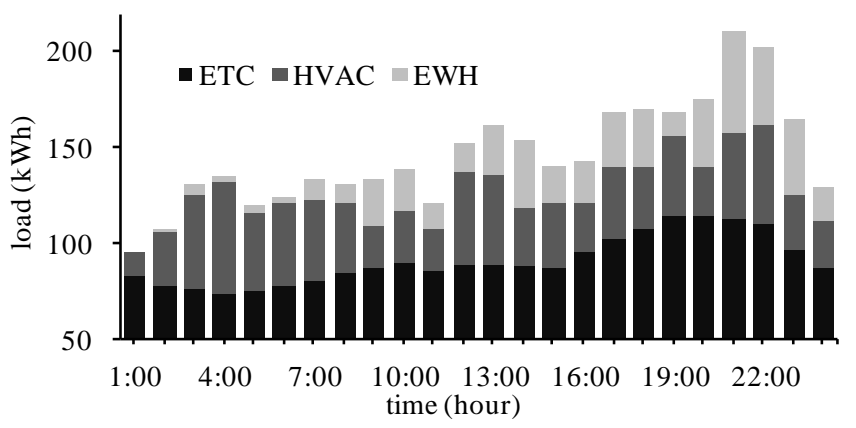

Fig. 4

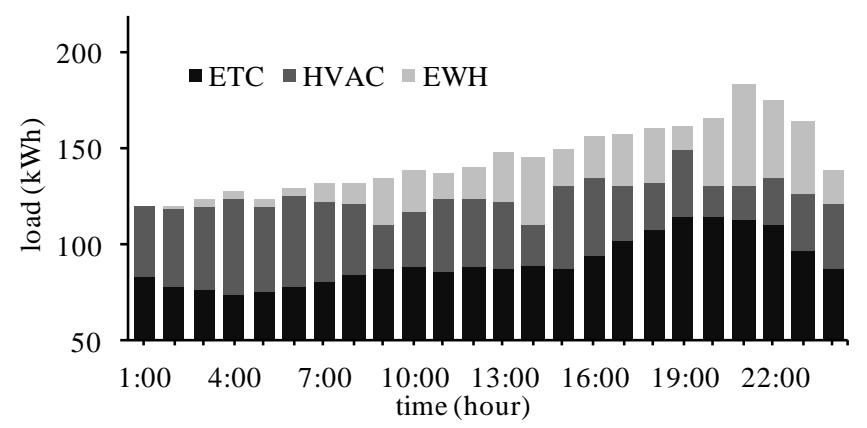

Fig. 5

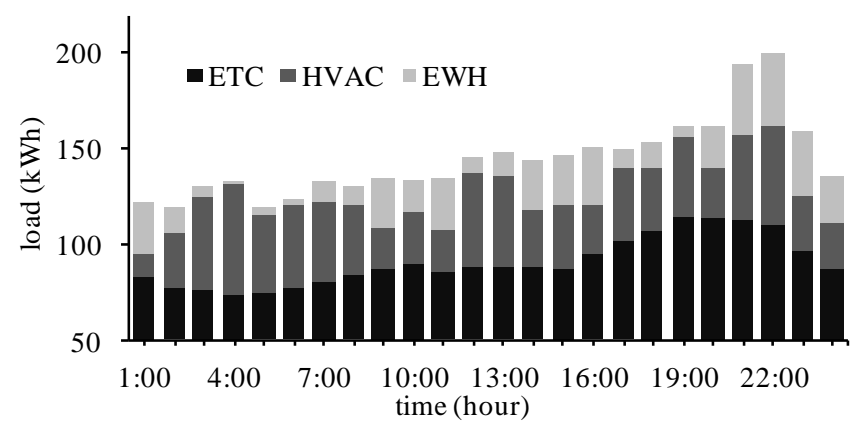

Fig. 6

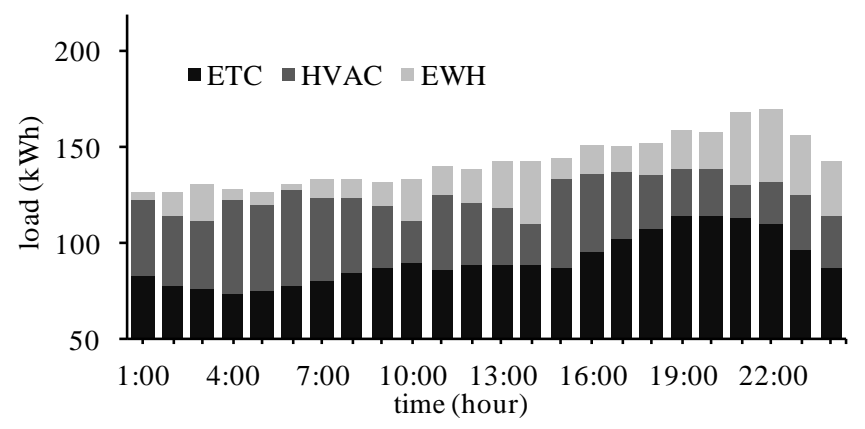

Fig. 7 


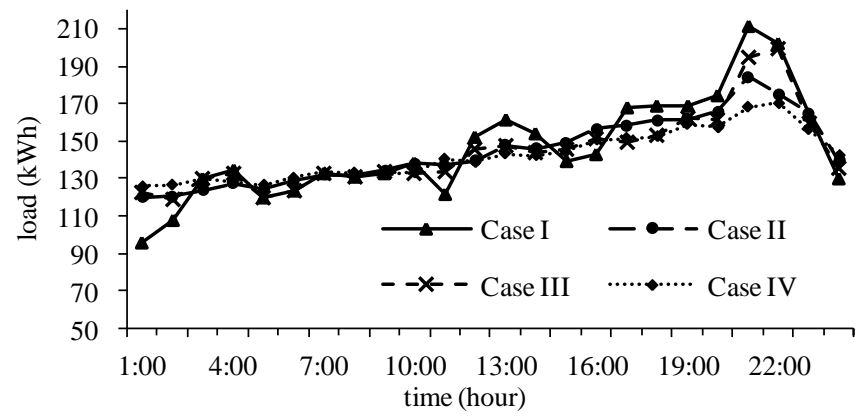

Fig. 8

Fig. 9
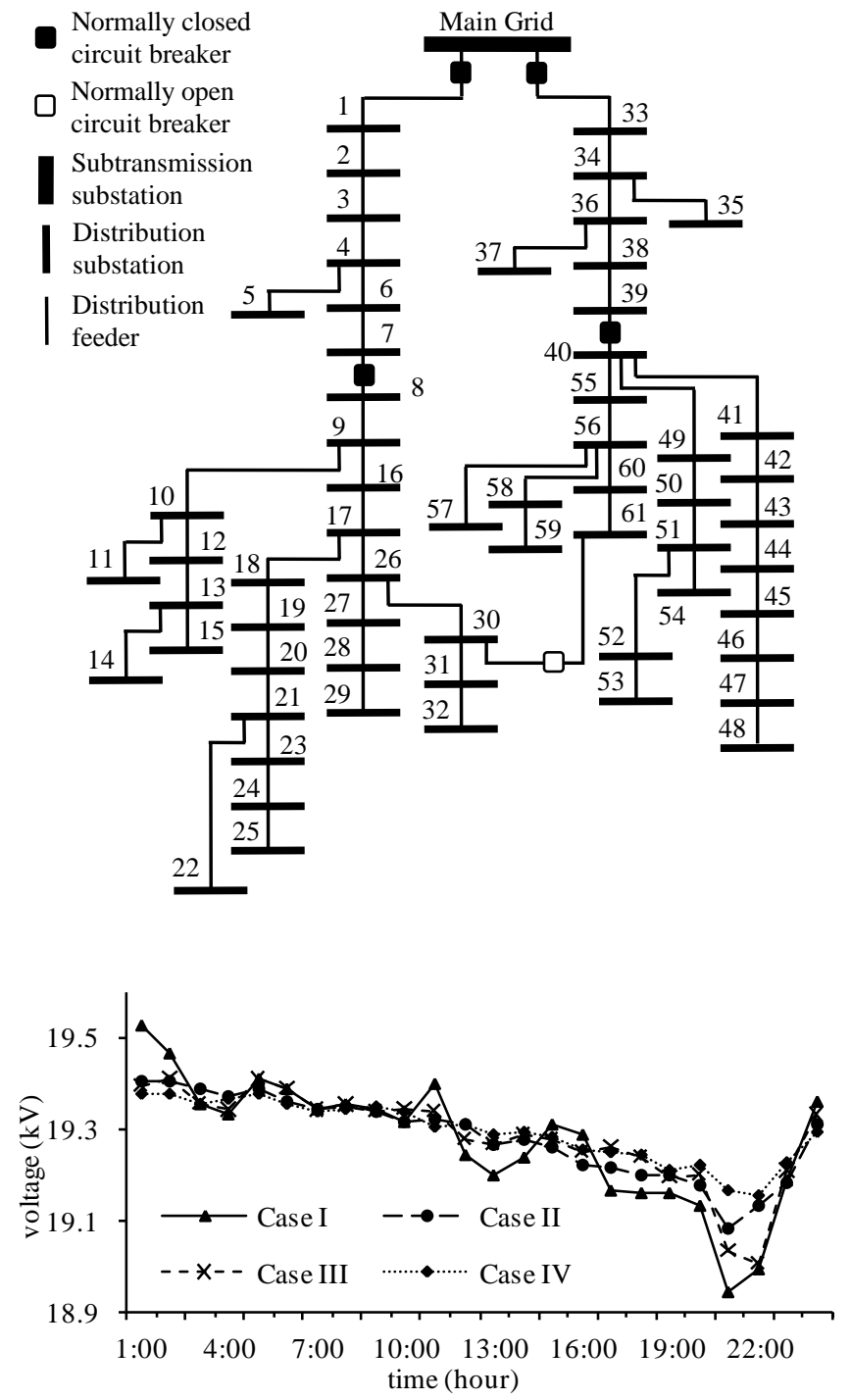

Tables: 
Table I

Table II

\begin{tabular}{|c|c|c|c|}
\hline$C_{n}\left(\mathrm{kJJ}^{\circ} \mathrm{C}\right)$ & $H_{n}^{i o}(\mathrm{~W} \rho \mathrm{C})$ & $H_{n}^{i g}\left(\mathrm{~W} /{ }^{\circ} \mathrm{C}\right)$ & $H_{n}^{i v}(\mathrm{~W} \rho \mathrm{C})$ \\
\hline $2485-3678$ & $43.5-64.4$ & $15-22.2$ & $73.5-108.8$ \\
\hline
\end{tabular}

Table III

\begin{tabular}{cc}
\hline$C_{w}\left(\mathrm{~kJ} / \mathrm{kg}^{\circ}{ }^{\mathrm{o}} \mathrm{C}\right)$ & $V_{n}$ (liter) \\
\hline 4.18 & 600 \\
\hline
\end{tabular}

\begin{tabular}{|c|c|c|c|c|c|}
\hline & Characteristic & Case I & Case II & Case III & Case IV \\
\hline & Peak load [kW] & 211.26 & 184.26 & 199.80 & 170.55 \\
\hline & Valley load [kW] & 95.66 & 120.41 & 119.04 & 126.04 \\
\hline & Load factor [\%] & 69.20 & 78.48 & 72.23 & 83.53 \\
\hline & Case I & Case II & Case III & \multicolumn{2}{|r|}{ Case IV } \\
\hline & $3508.61 \mathrm{kWh}$ & $3470.36 \mathrm{kWh}$ & $3463.61 \mathrm{kWh}$ & \multicolumn{2}{|r|}{$3418.98 \mathrm{kWh}$} \\
\hline
\end{tabular}

Table V

\begin{tabular}{ccccc}
\hline & & & & \\
Characteristic & Case I & Case II & Case III & Case IV \\
\hline \hline Total network losses [kWh] & 48.93 & 46.90 & 46.94 & 45.21 \\
\hline
\end{tabular}

Table VI

\begin{tabular}{ccccc}
\hline Index & Case I & Case II & Case III & Case IV \\
\hline \hline SAIFI [int./sub.-day] & 0.15134 & 0.14743 & 0.14819 & 0.14557 \\
\hline SAIDI [h/sub.-day] & 0.2688 & 0.2620 & 0.2609 & 0.2566 \\
\hline EENS [kWh] & 1802.91 & 1704.93 & 1699.11 & 1628.46 \\
\hline EIC [€] & 37239.86 & 35294.36 & 35340.67 & 33856.59 \\
\hline
\end{tabular}

Table VII

\begin{tabular}{ccccc}
\hline $\begin{array}{c}\text { Number of enrolled } \\
\text { consumers (out of 50) }\end{array}$ & $\begin{array}{c}\text { Peak load } \\
{[\mathbf{k W}]}\end{array}$ & $\begin{array}{c}\text { Load factor } \\
{[\mathbf{\%}]}\end{array}$ & $\begin{array}{c}\text { Total loss } \\
{[\mathbf{k W h}]}\end{array}$ & EENS [kWh] \\
\hline \hline $\mathbf{0}$ & 211.26 & 69.20 & 48.93 & 1802.91 \\
\hline $\mathbf{1 2}$ & 193.80 & 74.83 & 47.38 & 1719.07 \\
\hline $\mathbf{2 5}$ & 170.55 & 83.53 & 45.21 & 1628.46 \\
\hline $\mathbf{3 7}$ & 155.80 & 90.05 & 43.63 & 1555.01 \\
\hline $\mathbf{5 0}$ & 150.88 & 92.40 & 43.06 & 1528.15 \\
\hline
\end{tabular}

Table VIII

\begin{tabular}{cccccc}
\hline Allowed temperature band $\left[{ }^{\circ} \mathbf{C}\right]$ & Peak load & $\begin{array}{c}\text { Load fac- } \\
\text { tor [\%] }\end{array}$ & $\begin{array}{c}\text { Total loss } \\
{[\%]}\end{array}$ & EENS [\%] \\
\hline \hline Indoor & Hot water & 11.03 & 9.61 & 7.11 & 9.06 \\
\hline $\mathbf{2 0}-\mathbf{2 2}$ & $\mathbf{5 2}-\mathbf{5 8}$ & 19.27 & 20.71 & 7.60 & 9.68 \\
\hline $\mathbf{1 9}-\mathbf{2 3}$ & $\mathbf{5 0}-\mathbf{6 0}$ & 26.32 & 28.25 & 14.70 & 19.39 \\
\hline $\mathbf{1 8}-\mathbf{2 4}$ & $\mathbf{4 8}-\mathbf{6 2}$ & & &
\end{tabular}

Table IX

\begin{tabular}{ccccc}
\hline $\begin{array}{c}\text { Change in outdoor } \\
\text { temperature }\left[{ }^{\circ} \mathbf{C}\right]\end{array}$ & $\begin{array}{c}\text { Peak load } \\
{[\boldsymbol{\%}]}\end{array}$ & $\begin{array}{c}\text { Load factor } \\
{[\boldsymbol{\%}]}\end{array}$ & $\begin{array}{c}\text { Total loss } \\
{[\boldsymbol{\%}]}\end{array}$ & EENS [\%] \\
\hline $\mathbf{- 5}$ & 14.26 & 13.01 & 7.95 & 9.10 \\
\hline $\mathbf{- 2}$ & 17.24 & 18.01 & 6.85 & 8.10 \\
\hline--- & 19.27 & 20.71 & 7.60 & 9.68 \\
\hline $\mathbf{+ 2}$ & 23.28 & 25.89 & 9.38 & 12.40 \\
\hline $\mathbf{+ 5}$ & 18.31 & 18.45 & 8.87 & 13.11 \\
\hline
\end{tabular}

